\title{
Modeling Energy Demand Dependency in Smart Multi-Energy Systems
}

\author{
N. Neyestani ${ }^{1}$, Maziar Yazdani Damavandi ${ }^{1}$, Miadreza Shafie-khah ${ }^{1}$, \\ and João P.S Catalão ${ }^{1,2,3}$ \\ ${ }^{1}$ University of Beira Interior, Covilhã, Portugal \\ catalao@ubi.pt \\ ${ }^{2}$ INESC-ID, Lisbon, Portugal \\ ${ }^{3}$ IST, Univ. Lisbon, Portugal
}

\begin{abstract}
Smart local energy networks provide an opportunity for more penetration of distributed energy resources. However, these resources cause an extra dependency in both time and carrier domains that should be considered through a comprehensive model. Hence, this paper introduces a new concept for internal and external dependencies in Smart Multi-Energy Systems (SMES). Internal dependencies are caused by converters and storages existing in operation centers and modeled by coupling matrix. On the other hand, external dependencies are defined as the behavior of multi-energy demand in shifting among carriers or time periods. In this paper, system dependency is modeled based on energy hub approach through adding virtual ports and making new coupling matrix. Being achieved by SMESs, the dependencies release demandside flexibility and subsequently enhance system efficiency. Moreover, a test SMES that includes several elements and multi-energy demand in output is applied to show the effectiveness of the model.
\end{abstract}

Keywords: Dependency modeling, internal and external dependency, smart multi-energy system.

\section{Introduction}

Environmental issues as well as other critical issues such as global warming, emission of greenhouse gases and declining fossil resources have initiated trends towards sustainable development of energy and manipulation of various energy resources capacity. Sustainable energy development and preparation of future energy portfolio have to be in a manner that besides saving the environment does not interfere with the provision of future generation energy needs. In this regard, Smart Multi-Energy Systems (SMES) and consequently, Distributed Energy Resources (DERs) are two main tools for achieving these goals [1]. SMES accelerates the integration of various renewable-based resources and facilitates the participation of DERs in system operation. Development of DERs in SMES will provide bi-directional relation between distribution level and upstream grid which brings the advantages of network loss reduction and increases the reliability on demand side [2]. 
Moreover, DERs introduce dependency in both carrier and time domain to SMES through converters and storages. Distributed energy converters transform input energy carrier to output energy carrier and make mutually dependent carriers. In SMES with high penetration of DERs, these converters are spread all over the grid. As a result, network in various points has dependency between energy carriers [3]. Studies in such networks have to be simultaneously integrated, take into account all carriers' effects.

Two modeling approaches entitled "energy hub system" and "matrix modeling" were developed to model these dependencies ([4] and [5]). These models are based on some operation centers (energy hubs which mostly consist of cogeneration or trigeneration) and their interconnectors. The operation centers convert input energy carriers to the output required services and the interconnectors transmit energy carriers between operation centers. The energy flow in interconnectors has been investigated in [6] and [7] and new models have been proposed for integrated load flow of gas and electric networks. In [8], the energy hub system operation considering energy carriers price and operation objectives is surveyed. DERs impacts on energy hub system model are investigated in [9] and [10]. In these models the energy service vectors in the output (demand side) of operation centers are independent from each other.

Literature review reveals that researches could not successfully fulfill all the requirements of modeling dependency in demand side. Hence, this paper introduces a new concept of internal and external dependencies in SMES. Internal dependencies are assumed to be caused by converters and storages existing in operation centers and modeled by coupling matrix. External dependencies are defined as the behavior of multi-energy demand in shifting among carriers or time periods. This means that, in SMES, operation centers deliver energy to multi-energy demands which also consist of various end-use converters and storages [11]. It is presumed that demand consumes various energy carriers and one end-service may be achieved by multi input energy vectors (e.g. cooking in a household that can take place with either electric or gas oven). According to the intimate relation between multi-energy demands and SMES, it would be very complicated to model the multi-energy demands' elements inside an operation center coupling matrix. On the other hand, ignoring these external dependencies in decision making scheme of SMES may also reduce the accuracy of studies.

Two main approaches have been adopted for confronting with dependency of multi-energy demand in SMES: first group of researchers have neglected external dependencies and have modeled the network just before end-use (e.g. [4]); second group (e.g. [8] and [12]) have modeled the network with high resolution but in a very limited area such as a household and have modeled these dependencies in ultimate service level. In this method, although high resolution brings effects of multi-energy demand dependency, model is very complicated and the level of modeling should be limited.

This paper proposes a comprehensive framework for modeling both internal and external dependencies, which will help in investigating the role of multi-energy demand dependency. Multi-energy demands will bring higher levels of flexibility in the network. Modeling and manipulation of this flexibility will provide SMES operators with more freedom degrees and will reduce operation costs [13]. In this paper, energy hub approach is used for modeling internal and external dependencies 
in SMES. The comparison between the numerical results obtained from the proposed model and the ones from previous models demonstrates the usefulness of the proposed model.

In next section, a contribution to collective awareness systems is discussed. In Section 3, a comprehensive model is proposed for energy networks with multi-energy carriers' dependency. In Section 4, modeling the external dependencies of the network is described. Section 5 explains the results, and the conclusion is presented in Section 6.

\section{Contribution to Collective Awareness Systems}

Development in Information and Communication Technology (ICT) infrastructure unlocks new opportunities for SMES managers to merge independent administrative systems and to involve more players in system decision making procedure.

Considering emerging energy resources and increase of energy carriers' dependency, the needs for collaboration of independent energy system operators are inevitable. In this context, collective awareness systems can openly link independent energy entities in SMES and design the architecture of a new collaboration environment for the benefit of enterprise.

New models should be developed to implement collective awareness systems goals and to utilize huge amount of collected information for managing high interdependent SMES. These models consider energy infrastructure as integrated system which facilitates interaction between energy carriers. From modeling point of view, Energy $H u b$ system and Matrix Modeling, as explained in Section 1, are good examples for integrated models. Involving new smart elements and more participation of demand side are key factors for enhancing the level of smartness and awareness of these models.

Emerging multi-energy demands which can convert and store energy carriers, highlighted the role of demand side players in SMES decision making process. Modeling multi-energy demand behavior will involve new layer of players to the system which improves socio-technical aspect of integrated models.

\section{Comprehensive Smart Multi-Energy System Model}

Considering the presence of DERs in future SMES, energy carriers surely will be dependent to each other. Dependency in these forthcoming energy systems will be in a manner that studying some part of the network will not be possible nor correct without considering the dependencies of energy carriers in input or output of the model. Therefore, modeling the effects of DERs is essential in studies.

References [4] and [5] are main studies that have proposed a model for interdependent energy networks. The SMES in this paper has also been motivated by these references and is represented by a coupling matrix that converts the input energy carriers to output carriers. Each element of this matrix denotes the conversion of one carrier into another. Each element is composed of two parameter categories: first 
category of constant coefficients $\left(\eta_{\alpha}\right)$, which is dependent to physical characteristics of system and converters; second category includes decision making parameters $\left(v_{\alpha, t}\right)$ that indicate energy distribution between converters.

\subsection{Energy Converter Model}

In this paper, external dependencies are modeled through modules in output of the model. Output module indicates dependency of multi-energy demand that is not considered in the modeling process. This module represents the dependency between energy converters in the output with a function of carriers. This model shows that, multi-energy demand has the ability to receive definite amount of energy regardless of its carrier and convert it to its own required service. Dependency between outputs is added to demand vector through some lines, which itself increases the rows of coupling matrix. It is noteworthy that, the added lines do not represent actual outputs but virtually illustrates the dependency in output.

Hence, the output vector $(\boldsymbol{\lambda})$ in the proposed model can be divided into two sections: rows indicating independent output carriers $\left(\boldsymbol{\lambda}_{\mathrm{I}}\right)$ and rows introducing dependency in the output $\left(\boldsymbol{\lambda}_{\mathbf{D}}\right)$. With this regard, conversion function should have new rows rather than previous models, as shown by (1):

$$
[\lambda]=\left[\begin{array}{l}
\lambda_{I} \\
\lambda_{D}
\end{array}\right]=\left[\begin{array}{l}
\mathbf{C}_{I} \\
\mathbf{C}_{\mathbf{D}}
\end{array}\right] *[\mathbf{p}]
$$

where $\mathrm{C}_{\mathrm{I}}$ is the old coupling matrix that states the conversion of independent inputs to independent output and $C_{D}$ is the matrix showing share of independent inputs in providing dependent demand.

\subsection{Energy Storage Model}

As [6] has thoroughly explained, the role of energy storages can be modeled through some changes in the matrix. In the modified model, $\dot{E}$ is the change in stored energy and can be computed from (2) and (3). $\mathbf{S}$ is the coupling matrix of the storage and shows how changes in energy amount of storage will affect the system output. Equation (4) shows coupling matrix considering converters and storages model.

$$
\begin{aligned}
& \dot{E}_{\alpha, t}=E_{\alpha, t}-E_{\alpha, t-1} \approx e_{\alpha} Q_{\alpha} \\
& e_{\alpha}= \begin{cases}\eta_{\alpha, c h}^{\text {Sorage }}, & \text { if } Q_{\alpha} \geq 0 \quad(\text { Charge } / \text { Standby }) \\
1 / \eta_{\alpha, \text { lis }}^{\text {Storage }}, & \text { if } Q_{\alpha}<0 \quad \text { (Discharge) }\end{cases} \\
& {[\lambda]=\left[\begin{array}{ll}
\mathbf{C} & -\mathbf{S}
\end{array}\right] *\left[\begin{array}{l}
\mathbf{p} \\
\dot{\mathbf{e}}
\end{array}\right]}
\end{aligned}
$$

Considering external dependencies, new storage coupling matrix will have rows stating amount of changes in independent output based on changes in energy of storage: 


$$
\begin{gathered}
{[\lambda]=\left[\begin{array}{ll}
\mathbf{C} & -\mathbf{S}
\end{array}\right] *\left[\begin{array}{c}
\mathbf{p}_{\text {new }} \\
\dot{\mathbf{e}}
\end{array}\right]} \\
{\left[\begin{array}{l}
\lambda_{\mathrm{I}} \\
\lambda_{\mathrm{D}}
\end{array}\right]=\left[\begin{array}{cc}
\mathbf{C}_{\mathrm{I}} & \mathbf{S}_{\mathrm{I}} \\
\mathbf{C}_{\mathrm{D}} & \mathbf{S}_{\mathrm{D}}
\end{array}\right] *\left[\begin{array}{c}
\mathbf{p}_{\text {new }} \\
\dot{\mathbf{e}}
\end{array}\right]}
\end{gathered}
$$

Where $S_{I}$ and $S_{D}$ are matrices showing changes of independent and dependent outputs versus changes in the stored energy.

\section{Smart Multi-Energy System Operational Model}

Modeling external dependencies can considerably reduce system operation cost. Moreover, it brings more choices for system operators and planners while making it possible to utilize facilities of multi-energy demand in operation and planning studies. Fig. 1 illustrates a typical SMES that consists of CHP unit, auxiliary boiler, and heat storage and feeds a multi-energy demand. Input carriers of the system are electricity and gas while output carriers will be electricity, gas, and heat. External dependencies between gas and electricity carrier in this network is considered through a module in output. The mathematical model of this network is shown in (7):

$$
\left[\begin{array}{cccc}
v_{e, t}^{\text {out }} & v_{g, t}^{C H P} \cdot \eta_{e}^{C H P} \cdot v_{e, t}^{\text {out }} & 0 \\
0 & v_{g, t}^{C H P} \cdot \eta_{h}^{C H P}+v_{g, t}^{\text {boiler }} . \eta_{h}^{\text {boiler }} & 1 / e_{h} \\
0 & v_{g, t}^{\text {out }} & 0 \\
v_{e, t}^{d d} & v_{g, t}^{d d}+v_{e, t}^{d d} \cdot v_{g, t}^{C H P} . \eta_{e}^{C H P} & 0
\end{array}\right] *\left[\begin{array}{c}
p_{e, t} \\
p_{g, t} \\
\dot{h}_{t}^{\text {storage }}
\end{array}\right]=\left[\begin{array}{c}
L_{e, t} \\
L_{h, t} \\
L_{g, t} \\
L_{e g, t}
\end{array}\right]
$$

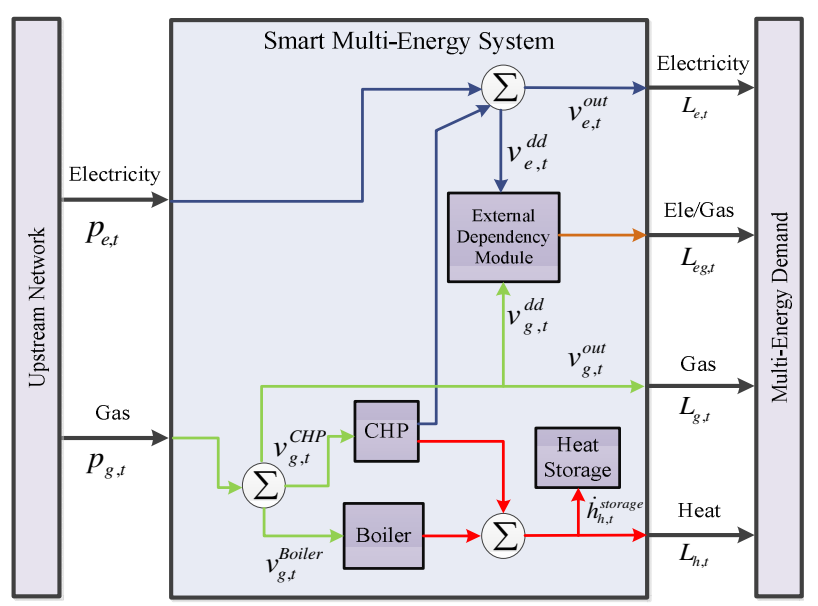

Fig. 1. A typical SMES model considering external dependency module 


\subsection{Objective Function and Operational Constraints}

The objective function is to minimize the cost of gas and electricity used as input. The nomenclature of the following equations is shown in Table 2 of Appendix.

$$
\operatorname{Min} \sum_{t}\left(p_{e, t} \pi_{e, t}+p_{g, t} \pi_{g, t}\right)
$$

The objective is minimized considering the system operational constraints as below:

1) Input carriers constraints: each energy carrier has a supply limit which may be due to energy amount in upstream or energy transmission limits.

$$
0 \leq p_{\alpha, t} \leq \bar{P}_{\alpha} \quad, \alpha \in e, g, e g
$$

2) Operational constraints of CHP unit: regarding manufacturing characteristics of CHP unit, they face limits in the amount of electrical power or heat output. Furthermore, based on (12), CHP unit should be operated in certain heat to power ratio.

$$
\begin{aligned}
& \underline{P}^{C H P} \leq p_{t}{ }^{C H P} \leq \bar{P}^{C H P} \\
& \underline{H}^{C H P} \leq h_{t}{ }^{C H P} \leq \bar{H}^{C H P} \\
& \gamma_{t}^{C H P}=h_{t}{ }^{C H P} / p_{t}^{C H P}
\end{aligned}
$$

3) Operational constraints of auxiliary boiler: heat energy output of auxiliary boiler has some limits in providing energy.

$$
\underline{H}^{\text {Boiler }} \leq h_{t}^{\text {Boiler }} \leq \bar{H}^{\text {Boiler }}
$$

4) Operational constraints of heat storage: Equations (14) and (15) indicate the limit for storing energy in heat storage.

$$
\begin{gathered}
\left|\dot{h}_{t}^{\text {storage }}\right| \leq r_{h}^{\text {storage }} \\
\underline{H}^{\text {Storage }} \leq h_{t}^{\text {storage }} \leq \bar{H}^{\text {Storage }}
\end{gathered}
$$

5) Decision-making variables constraints: decision-making variables will determine energy dispatch between various elements. These variables are system freedom degrees for optimization of least-cost operation procedure.

$$
\begin{gathered}
v_{e, t}^{d d}+v_{e, t}^{\text {out }}=1 \quad, 0 \leq v_{e, t}^{d d}, v_{e, t}^{\text {out }} \leq 1 \\
v_{g, t}^{C H P}+v_{g, t}^{\text {boiler }}+v_{g, t}^{d d}+v_{g, t}^{\text {out }}=1 \quad, 0 \leq v_{g, t}^{C H P}, v_{g, t}^{\text {boiler }}, v_{g, t}^{d d}, v_{g, t}^{\text {out }} \leq 1
\end{gathered}
$$

\subsection{Dependency Model of Multi-Energy Demand}

In a typical SMES, dependency due to multi-energy demand is modeled through a block connected to the output. The external dependency indicates that demand can utilize either electricity or gas carriers. The dependency is modeled through a block in the output, which is the function of dependencies between gas and electricity carriers. In order to deal with the dependency among carriers, two decision variables are used as: 
$v_{e}^{d d}, v_{g}^{d d}$ : Decision-making variables stating the share of dependent energy demand in output of each carrier (gas and electricity):

$$
f\left(v_{e, t}^{d d}, v_{g, t}^{d d}\right)=L_{e g, t}
$$

Dependency variables for external dependency will illustrate demand share; thus, it is necessary to balance them with some coefficients and then exploit them in the model. New decision variables in output show the share of each carrier in demand provision:

$$
\begin{gathered}
v_{e, t}^{d d-\text { new }}=v_{e, t}^{d d} \cdot\left(p_{e, t}+p_{g, t} \cdot v_{g, t}^{C H P} \cdot \eta_{e}^{C H P}\right) / L_{e g, t} \\
v_{g, t}^{d d-\text { new }}=v_{g, t}^{d d} \cdot p_{g, t, s} / L_{e g, t}
\end{gathered}
$$

\section{$5 \quad$ Numerical Results}

An SMES under study in this paper consists of CHP unit, auxiliary boiler, and heat storage. Inputs of this system are gas and electricity carriers while the outputs are electricity, gas, and heat. Detailed information of these elements is explained in Appendix Table 1.Moreover, information about multi-energy demand in base case and input energy carrier prices are depicted in Fig. 2 and Fig. 3. The external dependency in this study is hot water consumption of multi-energy demand, which can be supplied by both gas-fired and electrical heaters. Therefore, in a simple case heat consumption has been divided into two parts hot water and other consumption considering hot water as dependent output. The relation between electricity and gas carrier decision variable in the dependent input of multi-energy demand is shown with Eq. (21).

$$
v_{e, t}^{d d-n e w}+\eta_{g}^{d d} * v_{g, t}^{d d-n e w}=1
$$

In addition, in this study it is assumed that the system operator can control the gas and electricity dependent consumption through sending signals to load in smart environment and can benefit from the achieved flexibility. Four different levels of hot water consumption in the multi-energy demand and four different values for $\eta_{g}^{d d}$ (indicator of efficiency of gas-fired water heater) are assumed. Therefore, total 16 case studies are produced. In these cases, the amount of output energy consumption from SMES is the same whether ignoring the dependencies. For this reason, the dependency between electricity and gas is equally reduced from both output carriers' demand; as for gas share, it is adjusted based on the efficiency of gas-fired water heater.

As it is shown in Fig. 4, by increase in hot water consumption and utilization of this situation in equal levels of gas-fired water heater efficiency, total cost will decrease. On the other hand, by increasing controllable dependent consumption for the operator, operational freedom degree will increase, which results in lower system operation cost.

Fig. 5 shows prices versus output dependency amount. The re-decrease of costs after reaching a maximum amount should be highlighted. Due to even output energy amount of local energy network, by reducing in gas-fired water heater efficiency, the 
system will provide all dependencies through electricity carrier when their efficiency gets lower than electrical efficiency of CHP units in the system.

Therefore, with lower efficiency of multi-energy demand converters, demand requirements in output can be achieved by taking the benefits of less electricity with higher efficiency than the gas carrier and in a total view reducing the system operation cost. Fig. 6 and Fig. 7 depict the amount of input gas and electricity carriers when $\eta_{g}^{d d}=0.5$ for various levels of hot water consumption.

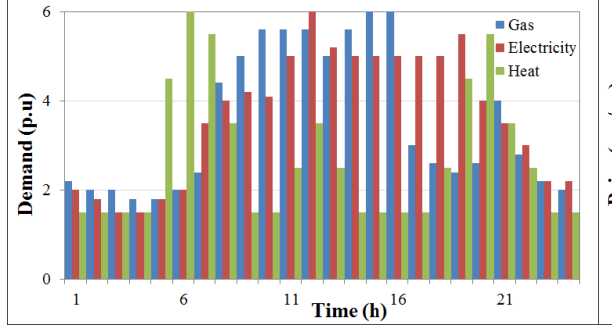

Fig. 2. Multi-energy demand data

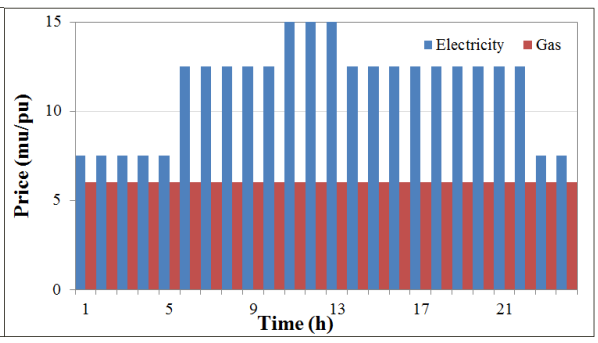

Fig. 3. Input energy carriers price data

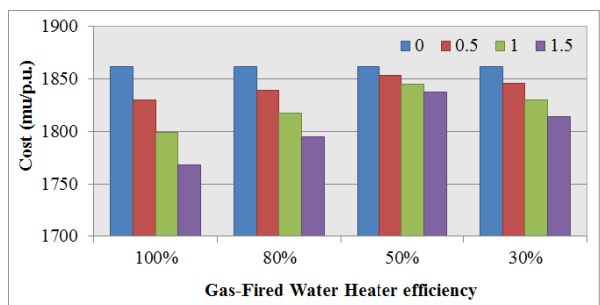

Fig. 4. System operation cost based on gasfired water heater efficiency

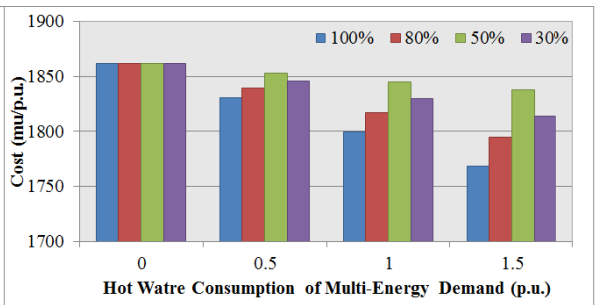

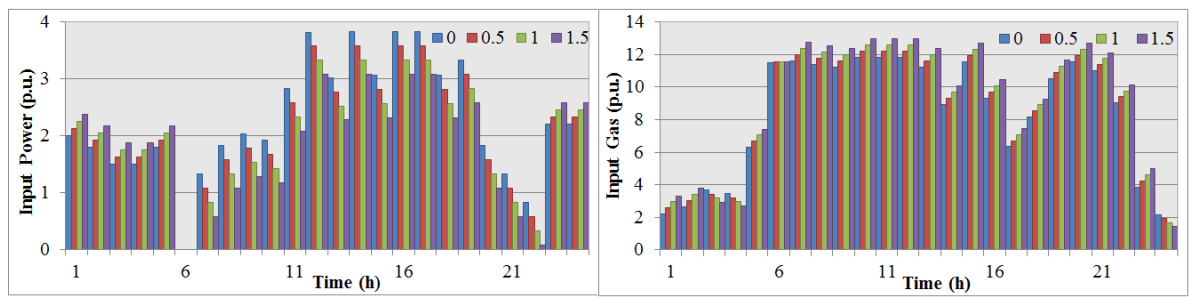

Fig. 6. Input power variation for various hot Fig. 7. Input gas variation for various hot water consumption with $\eta_{g}^{d d}=0.5$ water consumption with $\eta_{g}^{d d}=0.5$

As it can be observed, during hours 1-5, 23 and 24, when electricity price is lower, by increasing the level of flexibility tendency for electricity carrier consumption will increase while gas consumption shall decrease. However, during hours 6-22 the average electricity price is high, so the system operator prefers to provide hot water consumption through gas carrier and as a result reduces the total operation cost. 


\section{Conclusion}

The concept of internal and external dependencies in SMES was introduced. The external dependencies deal with the ability of multi-energy demand to implement its own converters. A new model based on energy hub approach was developed to represent internal and external dependency simultaneously. For assessing the efficiency of the model, an SMES with multi-energy demand in its output was considered. The numerical results confirmed that the external dependencies have a significant impact on the operation condition of the system. In addition, in the case of manageable external dependencies by increasing the dependency the operation cost will be decreased.

Acknowledgment. This work was supported by FEDER funds (European Union) through COMPETE and by Portuguese funds through FCT, under Projects FCOMP-01-0124FEDER-020282 (Ref. PTDC/EEA-EEL/118519/2010) and PEst-OE/EEI/LA0021/2013. Also, the research leading to these results has received funding from the EU Seventh Framework Programme FP7/2007-2013 under grant agreement no. 309048.

\section{References}

1. Alanne, K., Saari, A.: Distributed energy generation and sustainable development. Renewable and Sustainable Energy Reviews 10, 539-558 (2006)

2. Akorede, M.F., Hizam, H., Pouresmaeil, E.: Distributed energy resources and benefits to the environment. Renewable and Sustainable Energy Reviews 14, 724-734 (2010)

3. Chicco, G., Mancarella, P.: Distributed multi-generation: a comprehensive view. Renewable and Sustainable Energy Reviews 13, 535-551 (2009)

4. Krause, T., Andersson, G., Frohlich, K., Vaccaro, A.: Multiple-energy carriers: modeling of production, delivery, and consumption. Proc. IEEE 99, 15-27 (2011)

5. Chicco, G., Mancarella, P.: Matrix modelling of small-scale trigeneration systems and application to operational optimization. Energy 34, 261-273 (2009)

6. Arnold, M., et al.: Distributed predictive control for energy hub coordination in coupled electricity and gas networks. In: Intelligent Infrastructures, pp. 235-273. Springer (2010)

7. Damavandi, M.Y., Kiaei, I., Sheikh-El-Eslami, M.-K., Seifi, H.: New approach to gas network modeling in unit commitment. Energy 36, 6243-6250 (2011)

8. Bozchalui, M.C., et al.: Optimal operation of residential energy hubs in smart grids. IEEE Trans. Smart Grid 3, 1755-1766 (2012)

9. Kienzle, F., Ahcin, P., Andersson, G.: Valuing investments in multi-energy conversion, storage, and demand-side management systems under uncertainty. IEEE Trans. Sustainable Energy 2, 194-202 (2011)

10. Schulze, M., Friedrich, L., Gautschi, M.: Modeling and optimization of renewables: applying the energy hub approach. In: Proc. IEEE ICSET, pp. 83-88 (2008)

11. Mancarella, P., Chicco, G.: Real-time demand response from energy shifting in distributed multi-generation. IEEE Trans. Smart Grid (2013) (in press)

12. Houwing, M., Negenborn, R.R., De Schutter, B.: Demand response with micro-CHP systems. Proc. IEEE 99, 200-213 (2011)

13. Mancarella, P.: Smart multi-energy grids: concepts, benefits and challenges. IEEE PES General Meeting, 22-26 (2012) 


\section{Appendix. SMES Elements' Characteristics and Nomenclature}

Table 1. Data of SMES elements' Characteristics

\begin{tabular}{cccccccc}
\hline \multicolumn{2}{c}{ CHP Unit } & \multicolumn{2}{c}{ Auxiliary Boiler } & \multicolumn{2}{c}{ Heat Storage } \\
\hline $\begin{array}{c}\text { Output Energy } \\
(\mathrm{Min} / \mathrm{Max})\end{array}$ & $\eta_{e}^{\text {CHP }}$ & $\eta_{h}^{\text {CHP }}$ & $\begin{array}{c}\text { Output Energy } \\
(\mathrm{Min} / \mathrm{Max})\end{array}$ & $\eta_{h}^{\text {Boiler }}$ & $\operatorname{Tr}_{h}^{\text {Storage }}$ & $\begin{array}{c}\text { Stored Energy } \\
(\mathrm{Min} / \mathrm{Max})\end{array}$ & $\eta_{\alpha, c h}^{\text {Storage }}, \eta_{\alpha, \text { dis }}^{\text {Storag }}$ \\
\hline $0 / 5 \mathrm{pu}$ & 0.35 & 0.45 & $0 / 10 \mathrm{pu}$ & 0.9 & $3 \mathrm{pu}$ & $0.5 / 3 \mathrm{pu}$ & 0.9 \\
\hline
\end{tabular}

Table 2. Definition of Indices, Parameters, and Variables

\begin{tabular}{|c|c|c|c|}
\hline \multicolumn{4}{|c|}{ Subscripts } \\
\hline $\bar{e}$ & Electricity & Gas & Time \\
\hline$\underline{e g}$ & Dependent demand & Heat & Generic energy carriers \\
\hline \multicolumn{4}{|c|}{ Parameters and Variables } \\
\hline $\bar{E}$ & Energy stored & Efficiency & \\
\hline$H$ & Heat output & Electrical power & Decision variable \\
\hline$L$ & Energy demand & Heat to power ratio & Input energy carrier price \\
\hline$r$ & $\begin{array}{l}\text { Maximum charge and } \dot{\mathbf{e}} \\
\text { discharge rate of heat storage }\end{array}$ & $\begin{array}{l}\text { (column vector) changes } \dot{h} \\
\text { in stored energy }\end{array}$ & $\begin{array}{l}\text { Heat storage level difference in } \\
\text { two consecutive time intervals }\end{array}$ \\
\hline $\mathrm{Ar}$ & Inderlined (overlined) variable is us & d to represent the minimum $(\mathrm{m}$ & ximum) value of that variable. \\
\hline
\end{tabular}

